\title{
Secretory Head and Neck Neuroendocrine Tumour - A rare entity
}

\section{Tamar Saeed ${ }^{1}$, Michael Tadman $^{1}$, Aparna Pal $^{1}$, Samuel McKeith² ${ }^{2}$ Bahram Jafar-Mohammadi ${ }^{1}$}

${ }^{1}$ Oxford Centre for Diabetes, Endocrinology and Metabolism (OCDEM), ${ }^{2}$ ENT department, Oxford University NHS foundation trust

Introduction: Paragangliomas can derive from either parasympathetic or sympathetic paraganglia; the two types occur with similar frequency². The majority of parasympathetic ganglia-derived paragangliomas are located in the neck and skull base along the branches of the glossopharyngeal and vagus nerves ${ }^{3,4}$.They arise most commonly from the carotid body, less commonly from jugulotympanic and vagal paraganglia, and rarely, from the laryngeal paraganglia. The majority of paragangliomas arising within the skull base and neck region are not associated with catecholamine secretion; in various reports, up to 5 percent are symptomatic from hypersecretion ${ }^{5,7}$.

Presentation: We report a case of 43 year old gentleman who had surgery in 1998 for left glomus typanicum, leaving him with complete left lower facial nerve palsy and headaches. It is noted that he had labile blood pressure during surgery. There was gradual growth of the residual tumour associated with intermittent sweating.

In February 2016 he was referred to ENT for evaluation of his symptoms. In view of elevated metanephrines (Table 1) he was subsequently referred to endocrine service. In October 2016 he was confirmed to have a pathogenic mutation in succinate dehydrogenase $B(S D H B)$ gene [heterozygous for SDHB c.311delinsGG, P.(Asn104fs)]. A likely indolent left level IIb nodal metastasis was identified on FDG PET scan (Fig 1). The iodine-123-meta-iodobenzylguanidine (M IBG) scan demonstrated no increase uptake in the lesion (Fig 2). However, a skull base mass lesion and lymph nodes were avid on 68-GaDOTATATE PET CT(Fig 3).

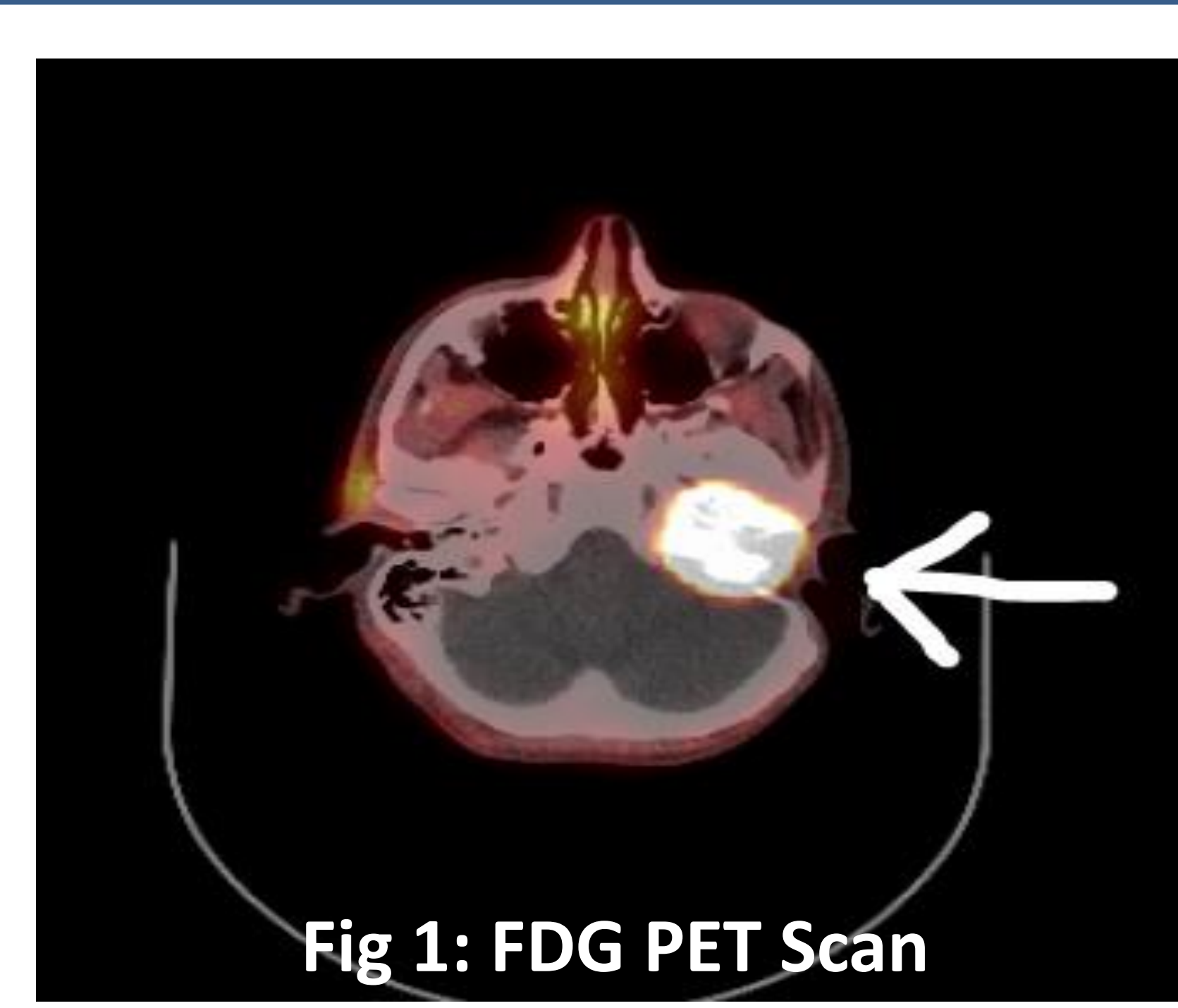

Table 1: Plasma Metanephrines - August 2016

\begin{tabular}{lll} 
Test & Result & Reference Range \\
\hline Methoxytyramine & $1061 \mathrm{pmol} / \mathrm{L}$ & $0-180 \mathrm{pmol} / \mathrm{L}$ \\
Metadrenaline & $259 \mathrm{pmol} / \mathrm{L}$ & $80-510 \mathrm{pmol} / \mathrm{L}$ \\
Normetanephrine & $1888 \mathrm{pmol} / \mathrm{L}$ & $120-1180 \mathrm{pmol} / \mathrm{L}$
\end{tabular}
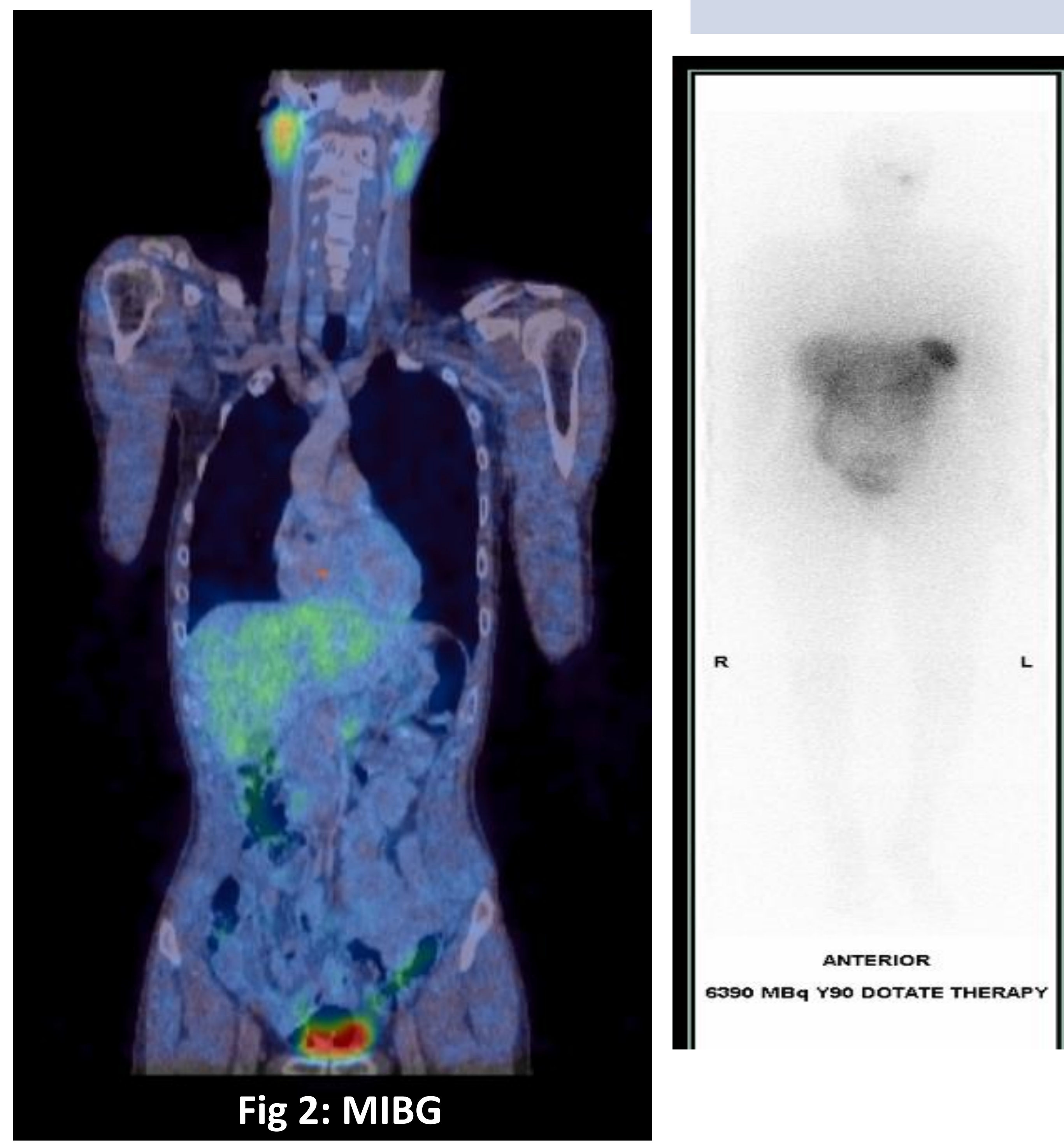

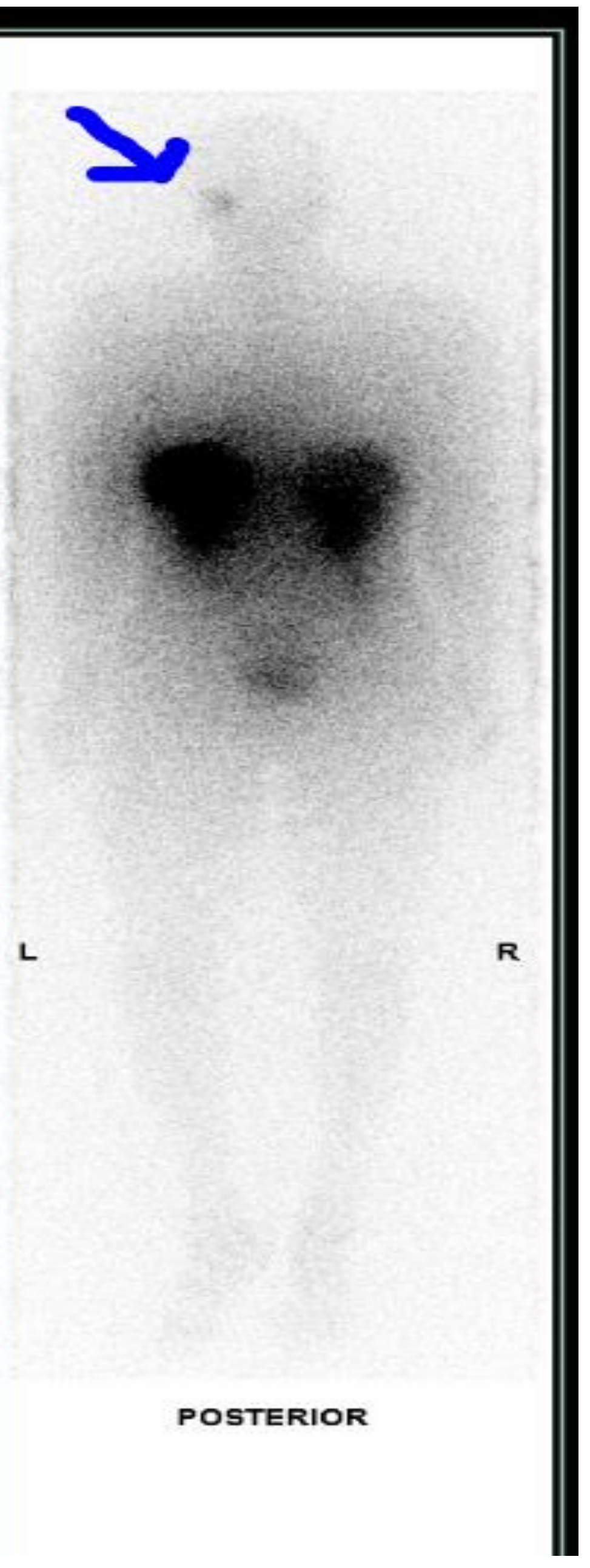

Fig 3: DOTATATE PET CT

Treatment: The patient was symptomatic and the biochemical markers confirmed the lesion to be functional (no other lesions were identified on scans). The utility of the Ga-68 DOTATE PET scan has provided a treatment option of Lutetium based Peptide Receptor Radionuclide Therapy (PRRT).

Conclusion: This case highlights that, though rare, head and neck paragangliomas can be secretory. Furthermore, the utilisation of appropriate functional imaging can be quite important in the treatment pathway. Genetic testing was carried out relatively late during the course of the management of this patient which may have given important information about likely course of the disease as well as imaging modalities that may have been useful in the detection of the disease.

References:

1. Pathology and Genetics of Tumours of the Endocrine Organs. WHO Classification of Tumours, DeLellis RA et al. 2004.

2. Welander $\mathrm{J}$ et al. . Genetics and clinical characteristics of hereditary pheochromocytomas and paragangliomas. Endocr Relat Cancer 2011

3. Kleinsasser O. Das Glomus laryngicum inferior. Ein bisher unbekanntes, nichtchromaffines Paraganglion vom Bau der sog. Carotisdrüse im menschlichen Kehlkopf. European Archives of Oto-Rhino-Laryngology 1964; 184:214.

4. Glenner GG, Grimley PM. Tumors of the Extra-Adrenal Paraganglion System. Bethesda, MD: Armed Forces Institute of Pathology, 1974.

5. van Duinen N, et al. Increased urinary excretion of 3-methoxytyramine in patients with head and neck paragangliomas. J Clin Endocrinol Metab 2010;

6. McNicol AM. Update on tumours of the adrenal cortex, phaeochromocytoma and extra-adrenal paraganglioma. Histopathology 2011;

7. Erickson D, et al. Benign paragangliomas: clinical presentation and treatment outcomes in 236 patients. J Clin Endocrinol Metab 2001

The Oxford Centre for Diabetes, Endocrinology and Metabolism
Oxford University Hospitals WHS

NHS Foundation Trust 